\title{
Editorial: Recent Advances on Communication Signal Processing and Networking
}

\author{
Fan -Yi Meng ${ }^{1}$ \\ Published online: 11 February 2020 \\ (C) Springer Science+Business Media, LLC, part of Springer Nature 2020
}

\section{Editorial:}

Due to the rapid developments in the field of communication technologies, signal processing and networking are paid great attentions in the next generation mobile networks to meet the demands of the massive connectivity and ubiquitous networking. 5G technologies are no longer limited to traditional contexts and platforms with huge potential. Several solutions have been proposed such as Massive MIMO, narrow band IoT (NB-IoT), NOMA, Zigbee, IEEE 802.11 and update of communication devices. Mobile networks have a large scale of potential applications like secure communication, cyber physical systems, multimedia communication and Internet of things environment. However, the application still faces challenges due to key limitations such as constant mobility, limited capability, restricted power, unguaranteed security, etc., which bring great challenges for technical research. The goal of this special issue is to bring the theoretical preparations and practical applications of research contribution in communication signal processing and networking.

This special issue features six selected papers with high quality. In the first article, "A Carrier Selection Method Based on Single RF Chain SM-OFDM Systems", a carrier selection method based on single RF chain SM-OFDM systems is proposed, a carrier grouping method for carrier selection and precoding are considered to reduce system complexity. Compared with the conventional SM-OFDM system, only single RF chain is used which avoids the inter-antennas interference and improves the energy efficiency. Moreover, the computational complexity of the system is greatly reduced.

The next article with the title "Antenna Selection in TDD Massive MIMO Systems" proposes an antenna selection method based on EE in uplink massive MIMO systems. Based on the channel state information (CSI) and the 2-

Fan -Yi Meng

fymenghit@gmail.com

1 Department of Microwave Engineering, Harbin Institute of Technology, Harbin, China

norm of channel matrix, the antennas with better CSI are selected for data transmission. By switching of the RF chain among multiple antennas dynamically over time, simulation results show that the EE can be enhanced compared with conventional massive MIMO system, and the trade-off between EE and spectral efficiency (SE) of the system can be achieved.

In recent years, relaying technique and non-orthogonal multiple access (NOMA) are introduced to visible light communication (VLC) system to improve the system performance. In "A Novel Relay-Assisted DCO-OFDM Green VLC System Based on NOMA", the authors studied a novel relay-assisted VLC system based on NOMA. All the sourceto-relay and relay-to-destination links in the proposed system use DCO-OFDM scheme to transmit information. The relay terminal serves two destination terminals at the same time. The signals in these two relay-to-destination links are superposed in the power domain based on NOMA. The total throughput with different parameters such as power allocation factors and DC bias is modeled and deduced in this paper.

The fourth article titled "Image Compression and Encryption Algorithm Based on Hyper-chaotic Map" is an extension of previous work. In the paper, aiming at overcoming defects which are low security properties, high costs of storage and transmission for exiting image encryption and compression algorithms. An algorithm which combined image compression and encryption based on hyper-chaotic map is proposed. In this algorithm, the original image is compressed by compression sensing (CS), and then the compressed image is encrypted through improved Arnold matrix transformation algorithm, Modular operation algorithm and combined the 3D hyper-chaotic map.

The authors of the fifth paper "Characteristics Analysis of the Fractional-Order Chaotic Memristive Circuit Based on Chua's Circuit" define a new fractional-order memristive circuit based on canonical Chua's circuit and Voltage-controlled memristor model. The fractional-order chaotic system is solved by conformable adomian decomposition method (CADM), and the complexity characteristics are analyzed 
through sample entropy (SampEn) algorithm. What's more, the chaos diagrams of complexity with the two parameters variation and the three parameters variation are analyzed.

The last article titled "A Multipath Routing Protocol with Load Balancing and Energy Constraining Based on AOMDV in Ad Hoc Network" designed a routing protocol based on load balancing with energy constraining, which selects nodes with lower queue length of MAC layer interface queue and higher residual energy to forward packets. The simulation results show that the performance of the improved protocol in terms of the number of energy exhausted node, average end-to-end delay and routing discovery frequency has been effectively improved.

Acknowledgements The guest editors are thankful to our reviewers for their effort in reviewing the manuscripts. We also thank the Edit-in-Chief, Dr. Imrich Chlamtac for his supportive guidance during the entire process. The special issue is sponsored by the Natural Science Foundation of Liaoning Province of China (Grant No. 20180550239), the National Natural Science Foundations of China (Grant No. 61601221), the Joint Foundations of the National Natural Science Foundations of China and the Civil Aviation of China (Grant No. U1833102), the China Postdoctoral Science Foundations (Grant Nos. 2015 M580425 and 2018 T110496), the Basic Scientific Research Projects of Colleges and Universities of Liaoning Province (Grant No. 2017 J045), the Provincial Natural Science Foundation of Liaoning (Grant No. 20170540060), the Scientific Research Projects in General of Liaoning Province (Grant No. L2015043), the Doctoral Research Startup Fund Guidance Program of Liaoning Province (Grant No. 201601280), as well as the Basic Scientific Research Projects of Colleges and Universities of Liaoning Province (Grant No. 2017 J046).
Publisher's note Springer Nature remains neutral with regard to jurisdictional claims in published maps and institutional affiliations.

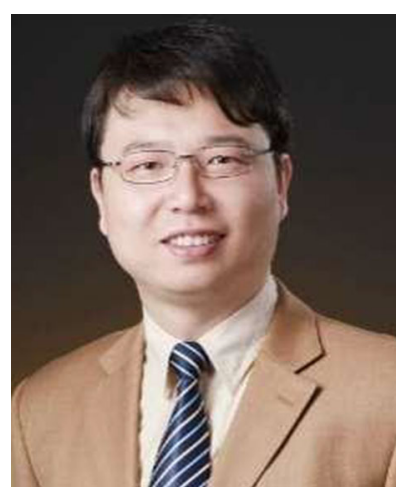

Prof. Fan-Yi Meng (S'07-M'09SM'15) received the B.S., M.S., and Ph.D. degrees in electromagnetics from the Harbin Institute of Technology, Harbin, China in 2002, 2004, and 2007, respectively. Since August 2007, he has been with the Department of Microwave Engineering, Harbin Institute of Technology, where he is currently a Professor. He has coauthored four books, 40 international refereed journal papers, over 20 regional refereed journal papers, and 20 international conference papers. His current research interests include antennas, electromagnetic and optical metamaterials, plasmonics, and electromagnetic compatibility (EMC). Dr. Meng was a recipient of several awards including the 2013 Top Young Innovative Talents of Harbin Institute of Technology, the 2013 CSTUniversity Publication Award, the 2010 Award of Science and Technology from the Heilongjiang Province Government of China, the 2010 "Microsoft Cup" IEEE China Student Paper Contest Award, two Best Paper Awards from the National Conference on Microwave and Millimeter Wave in China (2009 and 2007, respectively), the 2008 University Excellent Teacher Award of the National University of Singapore, the 2007 Excellent Graduate Award of Heilongjiang Province of China, and the Outstanding Doctor Degree Dissertation Award of the Harbin Institute of Technology. 